\title{
ARTICLE OPEN \\ Harmonization of Zika neutralization assays by using the WHO International Standard for anti-Zika virus antibody
}

\author{
Giada Mattiuzzo (iD ${ }^{1 *}$, Ivana Knezevic ${ }^{2}{ }^{2}$, Mark Hassall ${ }^{1}$, James Ashall ${ }^{1}$, Sophie Myhill ${ }^{1}$, Valwynne Faulkner ${ }^{1}$, Jason Hockley ${ }^{3}$, \\ Peter Rigsby $\mathbb{D}^{3}$, Dianna E. Wilkinson ${ }^{1}$, Mark Page $\mathbb{D}^{1}$ and the collaborative study participants
}

During outbreaks of emerging viruses, such as the Zika outbreak in 2015-2016, speed and accuracy in detection of infection are critical factors to control the spread of the disease; often serological and diagnostic methods for emerging viruses are not well developed and validated. Thus, vaccines and treatments are difficult to evaluate due to the lack of comparable methods. In this study, we show how the 1st WHO International Standard for anti-Zika antibody was able to harmonize the neutralization titres of a panel of serological Zika-positive samples from laboratories worldwide. Expression of the titres in International Unit per millilitre reduced the inter-laboratory variance, allowing for greater comparability between laboratories. We advocate the use of the International Standard for anti-Zika virus antibodies for the calibration of neutralization assays to create a common language, which will permit a clear evaluation of the results of different clinical trials and expedite the vaccine/treatment development.

npj Vaccines (2019)4:42

; https://doi.org/10.1038/s41541-019-0135-3

\section{INTRODUCTION}

Zika virus (ZIKV) is a flavivirus mainly transmitted by Aedes species mosquitoes. ZIKV was discovered in 1947 and was only known as a cause of sporadic mild disease in Africa and Asia., ${ }^{1,2}$ The first major outbreak occurred in 2007 in Yap Island with an attack rate as high as $70 \%$ of the population. ${ }^{3}$ In 2013 , during an outbreak in French Polynesia with a similarly high attack rate, the possible association with Guillain-Barré Syndrome was uncovered. ${ }^{4}$ The outbreak of ZIKV in the Latin American region in 2015-2016 was the largest recorded epidemic of Zika disease as of early 2019. Although the majority of Zika infections are asymptomatic or result in mild symptoms, the large number of cases in Latin America provided evidence for a correlation between Zika infection and increased cases of microcephaly and other neurological diseases., ${ }^{5,6}$ This association prompted the World Health Organization (WHO) to declare the outbreak a Public Health Emergency of International Concern (PHEIC) in February 2016. ${ }^{7}$ WHO called on the global research and product development (R\&D) communities to prioritize the development of vaccines together with improved diagnostics, and innovative vector control strategies for ZIKV R\&D. Although the PHEIC was declared over by the WHO DirectorGeneral in November 2016, ZIKV remains an enduring public health challenge requiring continued action, as new outbreaks may occur. ${ }^{8}$ Many uncertainties remain with regard to disease epidemiology and transmission, and, therefore, projecting the future evolution of the ZIKV epidemic and further spread based on the current knowledge remains difficult. In February 2018, WHO published "Zika Vaccine Development Technology Roadmap" to support development of a vaccine for outbreak use with the characteristics proposed within the Target Product Profile. ${ }^{9,10}$ In that context, standardization of virologic and immunologic assays for ZIKV vaccine development was identified as one of the priority areas. There is no licensed vaccine or treatment available, but development of these products is ongoing. According to the current knowledge on the transmission of ZIKV and experiences with past disease outbreaks, WHO has prioritized the development of vaccines suitable for use in an emergency or outbreak scenario. There are more than 40 vaccine candidates being developed using different platforms. Most of them are in the pre-clinical stage of development, but there are a few candidates which are the subject of clinical trials. ${ }^{11}$

Accurate diagnosis is essential to monitor and control the spread of ZIKV infection, as well as to provide guidance for pregnant women, or those planning to have children. ${ }^{12,13}$ Indeed, despite ZIKV infection occurring through the bite of infected mosquitoes of the genus Aedes, the virus can also be passed from mother to foetus and through exchange of body fluids. ${ }^{14-16}$ Molecular tests for ZIKV are the most reliable and specific, and there are several diagnostic tools authorized by the United States Food and Drug Administration under Emergency Use Authorization (EUA), while others are included in the WHO Emergency Use Assessment and Listing. ${ }^{13,17}$ However, ZIKV in blood/serum is short-lived (3-5 days), and up to 10 days in urine; ${ }^{18}$ therefore, a serological assay to measure immune exposure by antibody detection is suggested 7 days post onset of symptoms. ${ }^{13,17} \mathrm{~A}$ major problem with detection of anti-ZIKV antibodies is crossreactivity with other flaviviruses, such as dengue virus (DENV). ${ }^{19-21}$ Specifically, the envelope glycoprotein $E$ contain regions highly conserved between ZIKV and DENV, which are targets for binding and neutralizing antibodies. ${ }^{22}$ At the time of the outbreak in 2015/ 16 , the only authorized EUA test was the Zika Mac-ELISA (enzymelinked immunosorbent assay), which addresses the antibody cross-reactivity with other flaviviruses by requiring a 4-fold higher antibody titre in a plaque reduction neutralization assay against ZIKV than other flaviviruses to confirm infection. ${ }^{17}$ Recently, to avoid cross-reactivity, serological assays have been developed

\footnotetext{
${ }^{1}$ Division of Virology, National Institute for Biological Standards and Control (NIBSC), Blanche Lane, South Mimms, Potters Bar, Hertfordshire EN6 3QG, UK. ${ }^{2}$ Department of Essential Medicines and Health Products, World Health Organization, Avenue Appia 20, 1211 Geneva, Switzerland. ${ }^{3}$ Department of Biostatistics, National Institute for Biological Standards and Control (NIBSC), Blanche Lane, South Mimms, Potters Bar, Hertfordshire EN6 3QG, UK. A full list of consortium members and their affiliations appears at the end of the paper. *email: Giada.Mattiuzzo@nibsc.org
} 
targeting antibodies specific to the non-structural protein 1 (NS1), which is secreted by ZIKV-infected cells into the body fluids. ${ }^{23,24}$

Standardized, validated assays are of utmost importance not only for diagnosis of ZIKV infection and surveillance but also for vaccine development as anti-ZIKV antibody responses will most likely be used as immune markers for the evaluation of vaccine efficacy when large clinical trials with immunological/safety endpoints may be possible, but not clinical efficacy endpoints. ${ }^{25,26}$ Standardized assay methods have their limitations, however, due to variations in operator performance, different implementations among laboratories, and a lack of consistency over time as new refinements and technologies are introduced. Instead, the use of a biological standard that is common to all assays allows for calibration and harmonization of the data from different laboratories worldwide and bridges through advances in technology. The WHO International Standards (WHO ISs) are the highest order of reference materials and allow comparison of biological measurements (activity/potency) by defining an internationally agreed unit, the International Unit $\left(I^{27}\right)$. In-house or kit working standards should be calibrated using the WHO IS and the results reported in IU. Use of WHO IS has an impact on product development, for example, the majority of the molecular assays for hepatitis $\mathrm{C}$ virus (HCV) detection and quantification are calibrated to the WHO IS for HCV RNA; ${ }^{28}$ furthermore, the potency of Yellow Fever vaccines is expressed in $I^{29}$ by calibration of the assay to the 1 st International Standard for Yellow Fever vaccine. ${ }^{30}$

A multi-centre international collaborative study was organized to evaluate whether a reference preparation of sera or plasma from ZIKV-infected individuals could help harmonize serological assays for ZIKV antibody and the main outcomes of the study are presented in this article.

\section{RESULTS}

Results returned from neutralization assays

Nine laboratories $(2,3,7,8,11,12,13,14$, and 15; Table 1) returned data for live virus neutralization assays, and two laboratories (16 and 18) reported results for a reporter virus particle (RVP) neutralization assay. All neutralization assays were performed using a ZIKV Asian lineage, except laboratory 11a, which also included a ZIKV African lineage (MR766).

All laboratories identified correctly all the positive samples, these being either the pools of convalescent plasma/serum (samples S14, S48, S61, and S80-Table 2) or immunized transchromosomal (Tc) bovine immunoglobulins (samples S1 and S26). All the negative controls, plasma pool (sample S38), serum pool (sample S2), and naive Tc bovine immunoglobulin (sample S6) were negative in all the assays, except for that from lab 15, which indicated that sample S2 was a low positive. Seven out of 14 data sets did not show any cross-reactivity with the DENV reference preparations (samples S53, S79, and S93). Labs 3, 13, and 14c, however, scored either (lab 3) or both (labs 13 and 14c) of the DENV serotypes 1 and 2 (sample S53 and S79) positive; lab 7 identified DENV serotype 2 and 3 (samples S79 and S93) as positives. Labs 8 and 15 reported cross-reactivity with DENV serotype 3 . In all cases, the estimated potency of the DENV samples was lower than the ZIKV samples and therefore discriminatory. Table 3 shows the geometric means (GMs) of the results, which were provided as either median $50 \%$ plaque reduction neutralization titres $\left(\mathrm{PRNT}_{50}\right)$ or endpoint titre estimates.

Harmonization of the neutralization titres by the candidate IS Neutralization titres provided by the participant laboratories differed more than 100 -fold for some samples (Table 3, Fig. 1a). Variability across neutralization assays is intrinsic of this type of assay, where multiple factors play a role such as target cells, virus stock, timings, and other reagents used. Specifically, the different assay readouts, and their different ranges, have a critical impact; for example, lab 11 detected ZIKV infection by a reverse transcription-quantitative polymerase chain reaction (RT-qPCR) readout and has the highest titre values. To evaluate the effect of normalizing the data using a standard preparation, the results in Table 3 were expressed as relative to the values of sample S80 within each assay (Table 4). Sample S80, a pool of sera from Zika, confirmed cases returning to the UK, provided by the National Health Service Blood and Transplant (NHSBT) was chosen as the candidate International Standard because it was donated in a large quantity sufficient for the production of over 2000 ampoules and the material had not undergone any treatments prior to freeze-drying at National Institutes for Biologicals Standards and Control (NIBSC). Expression of the potency of each sample as relative to sample $\mathrm{S} 80$ reduced the variation between the labs for each positive sample (Table 5, Fig. 1b). In Table 5, the GM of each sample was calculated using the neutralization data from all labs except for lab 11a, where the potency values provided for each sample were considered outliers in comparison with the remaining data set. Since this was the only assay using ZIKV of African lineage, it was not possible in this study to determine whether the higher neutralization titres were lineage- or assay-dependent.

\section{Anti-ZIKV IgM detection in the pools of plasma/sera}

Seven laboratories $(1,2,6,10,14,17$, and 19-Table 1) processed the panel of samples in qualitative enzymatic immunoassays for the detection of anti-ZIKV immunoglobulin M (IgM). Most of the laboratories were able to detect specific lgM in known positive human convalescent samples S14, S48, S61, and S80. No signal was detected in the negative pools of plasma (sample S38) and sera (sample S2), or in the dengue reference preparations (samples S53, S79, and S93). The Tc bovine antibodies (samples S1, S6, and S26) were all negative as well. This was expected because the transgenic bovines do not generate human $\operatorname{lgM} .^{31}$ Laboratory $2 \mathrm{~d}$ found all the samples negative. Laboratory $6 \mathrm{a}$ scored positive the Tc bovine preparations and scored negative sample S80 (Supplementary Table 1). This result was due to the assay being under development at the time of this study, and an inefficient blocking method being used, which has now been improved to resolve these issues (personal communication with the study participant).

\section{Performance of the sample panel in other assays}

Anti-ZIKV binding immunoglobulin G (lgG) antibodies were detected in qualitative (assays 6b, 14b and 15a, Table 1) and quantitative enzymatic assays ( $1 a, 1 b, 2 c, 4,5 a$ and $5 c$, Table 1 ). Qualitative methods correctly identified all the positive samples except for the Tc bovine immunoglobulin (sample S1) from an animal immunized with plasmid DNA coding the E protein. Lab 14 only was able to detect this as a positive sample: this assay included multiple ZIKV antigens, while lab 6 and 15 assays are NS1-specific. All the control samples were correctly scored as negative, and no cross-reactivity was reported with the dengue samples (Supplementary Table 1).

Most of the quantitative ELISAs are specific for ZIKV NS1 and show no cross-reactivity with the dengue samples except for lab assay $1 \mathrm{a}$, which is an in-house assay using whole ZIKV as coating antigen. As expected, this assay was the only quantitative ELISA able to record a positive result for sample 1 , as this sample is derived from the Tc bovine immunized against ZIKV E protein only.

The data returned were reported using different readouts (Table 6) making comparisons between different ELISAs difficult; however, by reporting the results as a potency relative to sample S80, in a similar way to that used for the neutralization data, there was a good level of agreement between laboratories (Fig. 2). 
Table 1. Laboratory codes and assay methods

\begin{tabular}{|c|c|c|c|}
\hline Lab code & Assay method & Analyte (anti-)/ZIKV strain & Readout \\
\hline $1 \mathrm{a}$ & In-house indirect ELISA & $\begin{array}{l}\text { Whole virus inactivated/Asian strain- } \\
\text { PRVABC59 }\end{array}$ & OD \\
\hline $1 b$ & Commercial indirect ELISA (lgG) & NS1/African strain-MR766 & ELISA unit \\
\hline $1 c$ & Commercial capture IgM assay (qualitative) & NS1/not disclosed & POS/NEG \\
\hline $2 a$ & Neutralization assay & $\begin{array}{l}\text { Envelope protein/Asian strain-French } \\
\text { Polynesia (PF13/251013-18) }\end{array}$ & $\mathrm{TCID}_{50}$ \\
\hline $2 \mathrm{~b}$ & Neutralization assay & $\begin{array}{l}\text { Envelope protein/Asian strain-French } \\
\text { Polynesia (PF13/251013-18) }\end{array}$ & $\mathrm{PRNT}_{50}{ }^{\mathrm{a}}$ \\
\hline $2 c$ & Commercial indirect ELISA (lgG) & NS1/not disclosed & Relative unit $/ \mathrm{mL}$ \\
\hline $2 d$ & Commercial indirect ELISA (IgM) (qualitative) & NS1/not disclosed & POS/NEG \\
\hline 3 & Neutralization assay & Envelope protein/ Foraleza/2015 Brazil/2015 & $\mathrm{PRNT}_{50}{ }^{\mathrm{b}}$ \\
\hline 4 & Commercial indirect ELISA (lgG) & NS1/not disclosed & Relative unit/mL \\
\hline $5 a$ & In-house competitive ELISA & NS1/Asian lineage & OD \\
\hline $5 b$ & In-house indirect ELISA & NS1/Asian lineage & OD \\
\hline $6 a$ & $\begin{array}{l}\text { In development-commercial antigen-coated } \\
\text { nanoparticle agglutination (qualitative)-IgM }\end{array}$ & NS1/Suriname strain & POS/NEG \\
\hline $6 b$ & $\begin{array}{l}\text { In development-commercial antigen-coated } \\
\text { nanoparticle agglutination (qualitative)-IgG }\end{array}$ & NS1/Suriname strain & POS/NEG \\
\hline 7 & Neutralization assay & $\begin{array}{l}\text { Envelope protein H/PAN/2015/CDC-259359 } \\
\text { (Human/2015/Panama) }\end{array}$ & $\mathrm{PRNT}_{50}{ }^{\mathrm{b}}$ \\
\hline 8 & Neutralization assay & Envelope protein PRVABC59 & $\mathrm{MN}_{50}{ }^{\mathrm{a}}$ \\
\hline 9 & Surface plasmon resonance & Envelope protein-Brazil 2016 strain & Resonance units \\
\hline 10 & Capture IgM ELISA (qualitative) & Envelope protein-Asian lineage & POS/NEG \\
\hline $11 a$ & Neutralization assay & $\begin{array}{l}\text { Envelope-strains MR766 (Rhesus/1947/ } \\
\text { Uganda) }\end{array}$ & $\begin{array}{l}\text { Relative copy number by } \\
\text { RT-qPCR }\end{array}$ \\
\hline $11 b$ & Neutralization assay & Envelope-Asian lineage & $\begin{array}{l}\text { Relative copy number by } \\
\text { RT-qPCR }\end{array}$ \\
\hline 12 & Neutralization assay & Envelope-Paraiba/2015 & $\mathrm{PRNT}_{50}$ \\
\hline 13 & Neutralization assay & Envelope-PRVABC59 & $\mathrm{PRNT}_{50}$ \\
\hline $14 a$ & Capture IgM ELISA (qualitative) & Multiple antigens/HPF2013 & POS/NEG \\
\hline $14 \mathrm{~b}$ & Capture IgG ELISA (qualitative) & Multiple antigens/HPF2013 & POS/NEG \\
\hline $14 c$ & Neutralization assay & Envelope-Asian lineage (HPF2013) & $\mathrm{IC}_{50}{ }^{\mathrm{a}}$ \\
\hline $15 a$ & Neutralization assay (day 3 p.i.) & Envelope-PRVABC59 & $\mathrm{IC}_{50}$ \\
\hline $15 b$ & Neutralization assay (day 4 p.i.) & Envelope-PRVABC59 & $I C_{50}$ \\
\hline $15 c$ & Indirect epitope lockade of binding (qualitative) & NS1/Uganda and Suriname strains & POS/NEG \\
\hline 16 & Reporter virus particle neutralization assay & $\begin{array}{l}\text { Zika prM/E on a West Nile Virus } \\
\text { replicon system }\end{array}$ & $\mathrm{IC}_{50}^{\mathrm{a}}$ \\
\hline 17 & Commercial capture IgM/lgG (qualitative) & NS1/not disclosed & POS/NEG \\
\hline 18 & Reporter virus particle neutralization assay & $\begin{array}{l}\text { Protein C-prM-E from ZIKV strain SPH2015 on } \\
\text { reporter virus particles }\end{array}$ & $1 \mathrm{C}_{50}^{\mathrm{a}}$ \\
\hline 19 & Commercial IgM lateral flow (qualitative) & NS1/not disclosed & POS/NEG \\
\hline
\end{tabular}

EIA enzyme immunoassay, Neut neutralization assay, p.i. post infection, $P R N T$ plaque reduction neutralization test, $R T$-qPCR reverse transcription-quantitative polymerase chain reaction, TCID50 $50 \%$ tissue culture infectious dose, OD optical density, $P O S / N E G$ positive/negative, $M N_{50} 50 \%$ microneutralization titre, $I_{50}$ half-maximal inhibitory concentration

Note: Labs 2 and 4 used the same commercially available quantitative ELISA; labs 1c and 17 have the same commercially available qualitative ELISA

${ }^{\text {a }}$ Calculated as $\mathrm{IC} \mathrm{C}_{50}$ from inhibition curve

Inverse of the dilution, which achieved $50 \%$ reduction in plaques

However, only labs 2 and 4 produced a data set that allowed for the calculation of a relative potency by parallel line analysis.

Lab 9 analysed the panel of samples by surface plasmon resonance (SPR) targeting the E protein of ZIKV (Brazil 2016 isolate). The samples could be ranked similarly to their neutralization titres (Table 5) with the pools of sera/plasma producing higher resonance units (RU) than the human Tc bovine antibodies (comprising individual animals). All negative samples were below the cut-off limit, while dengue preparations scored above the cut- off limit of $30 \mathrm{RU}$, but at least five times lower than the weakest ZIKV-positive sample (sample S1, Supplementary Table 2).

Stability study

The stability of the candidate IS (sample S80) was assessed by accelerated thermal degradation testing. Ampoules were placed into storage at $45,37,20,4$, and $-20^{\circ} \mathrm{C}$, and retrieved at the following time points: 2 weeks, 1 month, 3 months, 6 months, and 
Table 2. Collaborative study samples

Sample code Sample description Donor

S1/TcEprot Purified human IgG from Tc bovine immunized with plasmid DNA encoding Zika $\mathrm{E}$ antigen

S2/HuNeg Human negative serum

S6/TcNeg Purified human IgG from naive Tc bovine, negative control

S14/HuUS Pool of 2 donors ZIKV-positive, plasma SD-extracted

S26/TcZIK Purified human IgG from Tc bovine immunized with whole killed Zika virus

S38/HuNeg Human plasma negative

S48/HuPR Pool of sera from 8 Zika-positive donors from Puerto Rico

S53/D1 Dengue serotype 1 International Reference Reagent

S61/HuCAR Pool of sera from 100 Zika-positive donors from the Caribbean SD-extracted

S79/D2 Dengue serotype 2 International Reference Reagent

S80/clS Candidate Zika International Standard (serum)

S93/D3

Dengue serotype 3 International Reference Reagent

Eddie J. Sullivan, SAB Biotherapeutics Inc., USA

NHSBT

Eddie J. Sullivan, SAB Biotherapeutics Inc., USA

Joseph Mauro, Boca Biolistics, Pompano Beach, FL, USA

Eddie J. Sullivan, SAB Biotherapeutics Inc., USA

NHSBT

Barney Graham and Julie Ledgerwood, Vaccine Research Centre, NIAD/NIH, USA

NIBSC

Richard Brindle, CARPHA, Trinidad and Tobago

NIBSC

Ines Ushiro-Lumb, NHSBT, Colindale, UK

NIBSC

CARPHA Caribbean Public Health Agency, NHSBT National Health Service Blood and Transplant, NIH National Institutes of Health, SD solvent detergent, Tc = trans-chromosomal

Table 3. Neutralization titres, reported by the participants, as the geometric mean of three independent experiments

\begin{tabular}{|c|c|c|c|c|c|c|c|c|c|c|c|c|c|c|}
\hline Sample & \multicolumn{14}{|c|}{ Laboratory } \\
\hline S1/TcEprot (+) & 509 & 1724 & 254 & 10 & 619 & 2528 & 2001 & 230 & 2259 & 460 & 308 & 182 & 2407 & 832 \\
\hline S2/HuNeg $(-)$ & $<10$ & ND & $<10$ & $<10$ & $<10$ & Neg & $\mathrm{Neg}$ & $<5$ & $<10$ & $<40$ & 16 & 14 & $<30$ & ND \\
\hline S6/TcNeg (-) & $<10$ & ND & $<10$ & $<10$ & $<10$ & Neg & $\mathrm{Neg}$ & $<5$ & $<10$ & $<40$ & $<10$ & $<10$ & $<30$ & ND \\
\hline S14/HuUS (+) & 7356 & 6062 & 4064 & 178 & 5812 & 317,650 & 19,610 & 2299 & 28,298 & 4268 & 3810 & 1726 & 3700 & 6709 \\
\hline S48/HuPR (+) & 2436 & 2714 & 1613 & 46 & 4975 & 346,681 & 10,358 & 1306 & 7833 & 4887 & 690 & 387 & 6818 & 4105 \\
\hline S53/D1 (-) & $<10$ & ND & 18 & $<10$ & $<10$ & Neg & Neg & $<5$ & 27 & 41 & $<10$ & $<10$ & $<30$ & ND \\
\hline S61/HuCAR (+) & 4299 & 2003 & 453 & 100 & 3114 & 88,555 & 2404 & 488 & 2801 & 1572 & 416 & 487 & 3515 & 3020 \\
\hline S79/D2 (-) & $<10$ & ND & $<10$ & 10 & $<10$ & Neg & Neg & $<5$ & 27 & 43 & $<10$ & $<10$ & $<30$ & ND \\
\hline S80/clS (+) & 4467 & 1477 & 640 & 68 & 2641 & 23,002 & 5443 & 939 & 7443 & 1094 & 725 & 951 & 3796 & 2590 \\
\hline
\end{tabular}

1 year and stored at $-20^{\circ} \mathrm{C}$ until assayed. The freeze-dried preparations were reconstituted as per instruction for use and tested concurrently in triplicate by in-house ELISA. Data are reported as relative to the baseline temperature $-20^{\circ} \mathrm{C}$ (Fig. 3). The relative potency after 1 year at each temperature did not differ from the baseline (e.g., 1 year at $45^{\circ} \mathrm{C}$ relative potency 0.97 ), suggesting that the material is sufficiently stable for long-term storage and can be shipped at ambient temperature.

\section{DISCUSSION}

The purpose of an IS is to harmonize data produced by laboratories worldwide. ${ }^{27}$ The concept of WHO ISs is based on the fact that laboratories use their own assays rather than one single assay. This means that the WHO ISs usually satisfy needs of users who are conducting different kind of assays. One of the objectives of a collaborative study such as this one is to demonstrate the suitability of the IS in the assays used in the collaborative study.

Through such multi-centre collaborative study, we have evaluated the ability of a pool of sera from ZIKV-infected patients to reduce the variability of the results from neutralization assays between 11 laboratories worldwide. Data returned from 14 methods showed a large magnitude of titres, which likely reflects the different methodologies used rather than performance of the assays (Table 3 ). All the assays were able to correctly identify the 
a

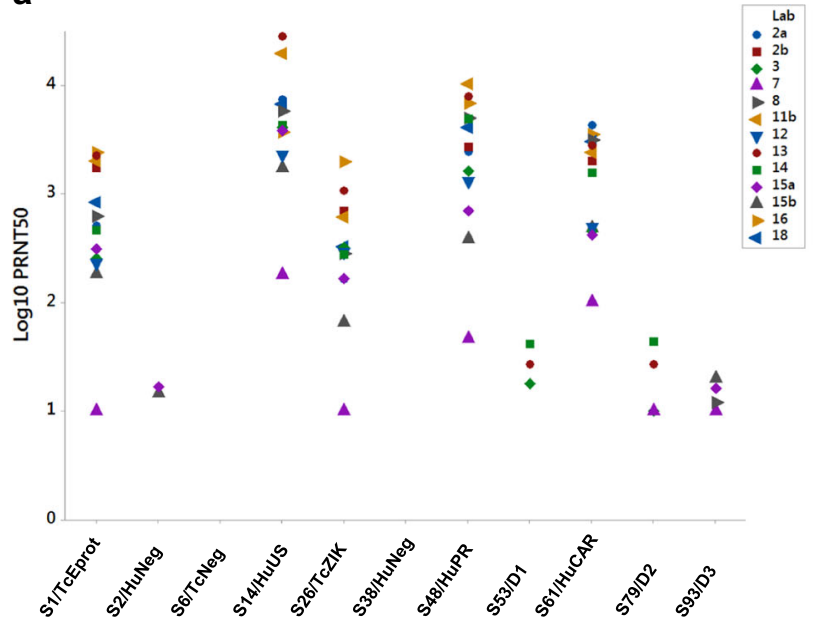

b

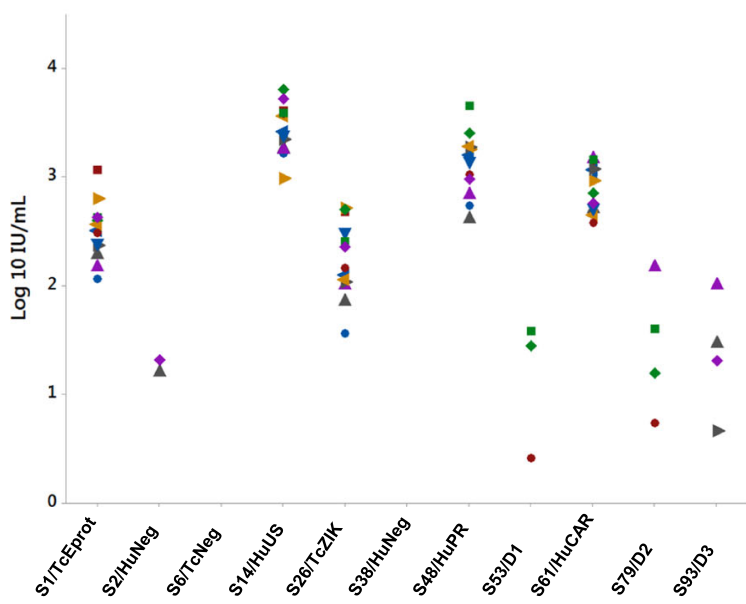

Fig. 1 Harmonization of the samples' potency when reported as relative to the candidate International Standard. a The geometric mean of neutralization titres for each sample as reported by the participants following three independent experiments (Table 3) are plotted; b neutralization titres were calculated relative to the candidate International Standard (sample S80), assuming an arbitrary value of 1000 IU/mL (Table 4). Samples S2, S6, and S38 are the negative controls; samples S53, S79, and S93 are the anti-dengue serum samples

Table 4. Neutralization titres expressed relative to sample 80 , assuming an arbitrary value of $1000 \mathrm{IU} / \mathrm{mL}$

\begin{tabular}{|c|c|c|c|c|c|c|c|c|c|c|c|c|c|c|}
\hline S2/HuNeg (-) & - & - & - & - & - & - & - & - & - & - & 21 & 16 & - & - \\
\hline 6/TcNeg (-) & - & - & - & - & - & - & - & - & - & - & - & - & - & - \\
\hline S14/HuUS (+) & 1647 & 4104 & 6350 & 1778 & 2201 & 15,566 & 3602 & 2448 & 3802 & 3902 & 5253 & 1815 & 975 & 2590 \\
\hline S38/HuNeg (-) & - & - & - & - & - & - & - & - & - & - & - & - & - & - \\
\hline S48/HuPR (+) & 545 & 1838 & 2520 & 681 & 1884 & 6874 & 1903 & 1391 & 1052 & 4468 & 952 & 406 & 1796 & 1585 \\
\hline S53/D1 (-) & - & - & 28 & - & - & - & - & - & 3 & 38 & - & - & - & - \\
\hline S61/HuCAR (+) & 962 & 1356 & 707 & 1468 & 1179 & 3240 & 442 & 520 & 376 & 1437 & 547 & 512 & 926 & 1166 \\
\hline S79/D2 (-) & - & - & - & 147 & - & - & - & - & 5 & 40 & - & - & - & - \\
\hline S93/D3 (-) & - & - & - & 100 & 5 & - & - & - & - & - & 20 & 29 & - & - \\
\hline
\end{tabular}

positive samples, and only one laboratory identified the negative serum preparation (sample S2) as a false positive. Some assays showed cross-reactivity with the Dengue reference preparations in the panel; however, this may reflect the presence of crossneutralizing antibody epitopes in the ZIKV E protein. ${ }^{22}$ Indeed, there was no cross-reactivity observed in those binding assays targeting NS1 protein only (Table 6).

Neutralization titres of the collaborative study samples as reported by the participants differed by up to 100 -fold; however, normalization by the candidate IS (sample S80) produced more comparable results with a reduction of inter-laboratory variance (Tables 4, 5 and Fig. 1). Similar results have been obtained in the collaborative study for the establishment of the 1st WHO IS for Ebola antibody, ${ }^{32}$ but inter-lab variability is often specific to the assay target and such comparisons are not easily made. Interestingly, the only method using ZIKV of African lineage produced the highest neutralization titres, about 10 times higher than the other assays (Table 4). As only one laboratory performed the neutralization assay with an African isolate, using a detection method (RT-qPCR) not comparable with other neutralization assays in this collaborative study, no conclusions can be inferred from these data. Although the ZIKV African lineage is more virulent than the Asian lineage in vitro and in animal models, ${ }^{33-36}$ a previous study has not reported a difference in neutralizing antibodies between the two lineages in convalescent human serum or plasma. ${ }^{37}$ Further investigations are clearly needed to resolve this issue so as to understand the possible reasons for the difference in titres.

The candidate IS and the panel of samples were further characterized in other serological methods such as ELISA and SPR, where the samples ranked similarly to the neutralization results. Importantly, expressing the ELISA results as relative to sample S80 allowed the comparison of results from different kits and in-house methods (Table 6 and Fig. 2). Unfortunately, it was not possible to assign the candidate IS as a calibrator for ELISA methods from this collaborative study. Only two sets of data from labs 2 and 4 were 
Table 5. Reduction in the inter-laboratory variance by expressing the neutralization titres as relative to the candidate International Standard

\begin{tabular}{llllll}
\hline & \multicolumn{2}{l}{ GM } & & & \multicolumn{2}{l}{ GCV } \\
\cline { 2 - 3 } \cline { 6 - 6 } & NT & IU/mL & & Raw data & Relative to sample 80 \\
\hline S1/ TcEprot (+) & 481 & 318 & & $337 \%$ & $84 \%$ \\
S2/HuNeg (-) & 15 & 18 & & \\
S6/TcNeg (-) & & & & \\
S14/HuUS (+) & 4290 & 2756 & & $240 \%$ & $70 \%$ \\
S26/TcZIK (+) & 268 & 173 & & $274 \%$ & $126 \%$ \\
S38/HuNeg (-) & & & & \\
S48/HuPR (+) & 2023 & 1339 & & $343 \%$ & $93 \%$ \\
S53/D1 (-) & 27 & 14 & & $52 \%$ & $338 \%$ \\
S61/HuCAR (+) & 1222 & 808 & & $217 \%$ & $62 \%$ \\
S79/D2 (-) & 23 & 32 & & $108 \%$ & $327 \%$ \\
S80/cIS (+) & 1511 & $1000^{\text {a }}$ & & $243 \%$ & \\
S93/D3 (-) & 14 & 23 & & $35 \%$ & $257 \%$ \\
\hline
\end{tabular}

Geometric mean value of the neutralization titres (NT) as reported by the participants or expressed as relative $(\mathrm{IU} / \mathrm{mL})$ to sample $\mathrm{S} 80$ with an assigned potency of $1000 \mathrm{IU} / \mathrm{mL}$; expected status of each sample is indicated in the sample name as ZIKV antibody positive (+) or negative (-) ${ }^{\text {a Assigned value }}$

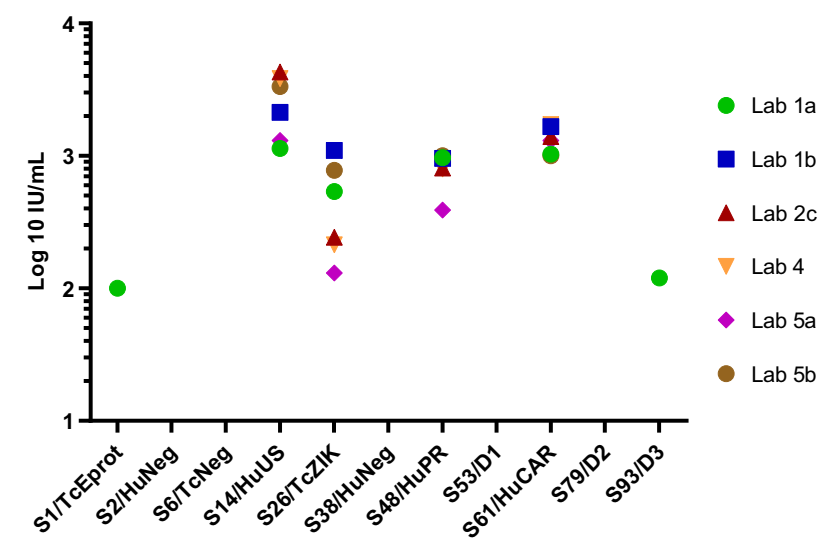

Fig. 2 ELISA potencies calculated relative to the candidate International Standard. The samples were analysed using commercial and in-house quantitative assays as described in Table 6 . The potencies in $\mathrm{IU} / \mathrm{mL}$ were calculated from the geometric mean of three independent assays of the data expressed relative to the value of sample S80, which was assigned an arbitrary value of $1000 \mathrm{IU} / \mathrm{mL}$ Only the estimates calculated for labs $2 c$ and 4 were valid by parallel line analysis

statistically valid by parallel line analysis (see methods), and therefore it was not possible to demonstrate that the candidate IS was able to reduce inter-laboratory variance. Suitability of the IS for anti-ZIKV antibodies in ELISA methods would require an additional collaborative study with a sufficient number of laboratories conducting quantitative ELISA and fulfilling statistical criteria for this kind of study.

It is important to highlight that all pools of convalescent serum/ plasma in this study had some reactivity to DENV. Anti-DENV reference materials from NIBSC (samples S53, S79, and S93) were included in the study to assess assay specificity. Although crossreactivity against DENV of neutralizing antibodies elicited by ZIKV infection has been reported, the lower titre (4-10-fold) allows for discrimination of the ZIKV infection in a DENV-immune patient. ${ }^{38}$ In this study, all the assays were able to differentiate ZIKV
Table 6. Quantitative ELISA data

\begin{tabular}{lllllll}
\hline & $1 \mathrm{a}^{\mathrm{a}}$ & $1 \mathrm{~b}^{\mathrm{b}}$ & $2 \mathrm{c}^{\mathrm{b}}$ & $4^{\mathrm{b}}$ & $5 \mathrm{a}^{\mathrm{c}}$ & $5 \mathrm{~b}^{\mathrm{c}}$ \\
\hline S1/TcEprot (+) & 0.1 & - & - & - & - & - \\
S2/HuNeg (-) & - & - & - & - & - & - \\
S6/TcNeg (-) & - & - & - & - & - & - \\
S14/HuUS (+) & 1.1 & 58.4 & 1365.0 & 1048.1 & 10.0 & 10.0 \\
S26/TcZIK (+) & 0.5 & 30.0 & 77.2 & 58.2 & 1.0 & 2.3 \\
S38/HuNeg (-) & - & - & - & & - & - \\
S48/HuPR (+) & 1.0 & 26.2 & 257.8 & 218.0 & 3.0 & 3.0 \\
S53/D1 (-) & - & $\mathrm{N} / \mathrm{T}$ & - & & - & - \\
S61/HuCAR (+) & 1.0 & 45.6 & 446.5 & 472.4 & 10.0 & 3.0 \\
S79/D2 (-) & - & $\mathrm{N} / \mathrm{T}$ & - & & - & - \\
S80/cIS (+) & 1.0 & 27.3 & 318.1 & 273.6 & 7.7 & 3.0 \\
S93/D3 (-) & 0.1 & $\mathrm{~N} / \mathrm{T}$ & - & - & - & - \\
\hline
\end{tabular}

$\mathrm{N} / \mathrm{T}=$ not tested; expected status of each sample is indicated in the sample name as ZIKV antibody positive (+) or negative (-)

an-house assay, potency relative to internal standard

${ }^{\mathrm{b}}$ Commercial assay, ELISA relative unit based on kit standard-labs $2 \mathrm{c}$ and 4 used the same kit

In-house assay, inverse of the last positive dilution

antibodies in the samples provided, confirming that the candidate International Standard (sample S80) contains ZIKV-specific antibodies. However, due to the presence of cross-reactive anti-DENV antibodies, the IS for anti-ZIKV antibodies cannot be used for the validation of specificity of ZIKV serological assays.

The outcome of this study was that the NHSBT pool of convalescent serum sample (Sample Code S80) was established by the WHO Expert Committee on Biological Standardization in October 2018 as the 1st WHO IS for anti-Asian lineage ZIKV antibody to be used in the standardization and assessment of neutralization assays with an assigned unitage of $250 \mathrm{IU}$ per ampoule. $^{39}$ The IS is available from the NIBSC catalogue (www. nibsc.org/), code 16/352, in ampoule numbers anticipated to be available for 5-10 years; stability studies have shown that the preparation is also stable for long-term storage. Furthermore, sample S14, the pool of convalescent plasma (S14) from Boca Biolistics, is also available in the NIBSC on-line catalogue (code 16/ 320 ) as a working reagent calibrated to the 1 st WHO IS in this collaborative study. The calculated value of $16 / 320$ is $2756 \mathrm{IU} / \mathrm{mL}$, with 95\% confidence limits of 2003 to 3792.

This study has shown how normalization of the data from different assays by the IS allows for comparison and harmonization of results from laboratories worldwide. The use of the IS reduced the inter-laboratory variation, albeit there is still up to a 10 -fold difference in the titres reported in $\mathrm{IU} / \mathrm{mL}$. Use of standardized methods with a validated standard operation procedure and sharing of the same reagents could be a further step to reduce inter-laboratory variations; however, this is beyond the scope of this work and could be evaluated by the scientific community and regulatory bodies working on Zika. Nevertheless, calibration of assays using the IS for ZIKV antibodies will allow for the consistency of antibody measurements among laboratories, the comparative analysis of candidate vaccines in clinical trials, which aim to determine protective antibody levels in human or animal models, leading to a batch/lot release specification; therefore, resulting in expedited licensing and subsequent availability of vaccines.

In conclusion, WHO standardization activities led by NIBSC resulted in the development and establishment of the 1st IS for anti-Asian lineage ZIKV antibody. This is an important achievement for the scientific community across ZIKV diagnostics, 
a

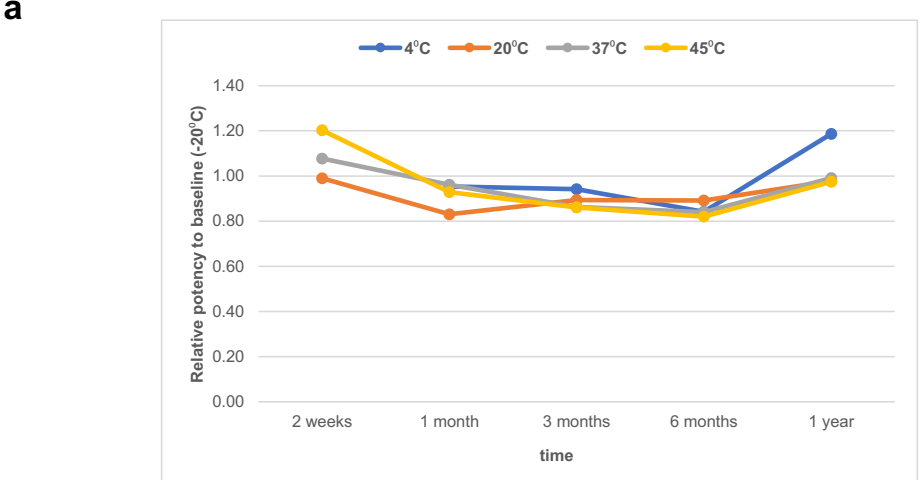

b

\begin{tabular}{|ccccc|}
\hline TimelTemperature & $\mathbf{4}^{\mathbf{0}} \mathbf{C}$ & $\mathbf{2 0}{ }^{\circ} \mathbf{C}$ & $\mathbf{3} \mathbf{7}^{\circ} \mathbf{C}$ & $\mathbf{4 5}^{\circ} \mathbf{C}$ \\
\hline 2 weeks & $0.95(0.92-0.99)$ & $0.99(0.94-1.04)$ & $1.08(1.03-1.13)$ & $1.20(1.16-1.24)$ \\
1 month & $0.94(0.90-0.98)$ & $0.83(0.78-0.88)$ & $0.96(0.91-1.02)$ & $0.93(0.82-1.05)$ \\
3 months & $0.99(0.96-1.02)$ & $0.89(0.82-0.98)$ & $0.86(0.80-0.92)$ & $0.86(0.83-0.89)$ \\
$\mathbf{6}$ months & $0.84(0.79-0.89)$ & $0.89(0.84-0.94)$ & $0.84(0.79-0.89)$ & $0.82(0.77-0.88)$ \\
1 year & $1.19(1.12-1.25)$ & $0.98(0.93-1.03)$ & $0.99(0.91-1.07)$ & $0.97(0.92-1.04)$ \\
\hline
\end{tabular}

Fig. 3 Thermal degradation assessment of the candidate International Standard for ZIKV antibody. Freeze-dried ampoules of sample S80 (NIBSC code 16/352) were stored at five different temperatures $\left(45,37,20,4\right.$, and $\left.-20^{\circ} \mathrm{C}\right)$. At each time point, three vials were retrieved and reconstituted with $0.25 \mathrm{~mL}$ of molecular grade water. Each vial was assessed in triplicate in the in-house ELISA. Data are reported relative to the storage temperature $-20^{\circ} \mathrm{C}$; a the graph shows the variation in potency against time; $\mathbf{b}$ the bottom table contains the mean value of three independent experiments of the relative potency with the $95 \%$ confidence limit within parentheses

prevention, and treatment. It is expected that the abovementioned standard will be used in the clinical development of ZIKV vaccines with the aim of reporting results of the assessment of the immune response in IUs. There have been examples of missed opportunities in using the ISs for development of new vaccines. There are several reasons, but the most important are: (1) lack of understanding among principal investigators of the benefits that the IS provide in the interpretation of data from clinical trials and (2) misunderstandings about assay standardization that less experienced vaccine developers are facing when establishing testing procedures for vaccines under development. Multiple vaccine candidates have shown robust protection against ZIKV challenge in animal models, but the demonstration of the protection in humans remains a challenging task. For the comparability of clinical trial results, it is critical to use WHO IS as a basis for assay standardization and optimization. Correct interpretation of the results from clinical trials is one of the essential elements to assure quality, safety, and efficacy of vaccines and diagnostics. Feedback from users will help WHO and its Collaborating Centre, NIBSC, to advance the further use of this standard as well as the development of other standards and reagents that may improve standardization of assays used in the clinical evaluation of Zika vaccines.

\section{METHODS}

\section{Participants}

Nineteen participants with an anti-ZIKV antibody assay in use in their laboratories completed the study. Prerequisites for participation in the collaborative study are: (1) availability of reliable assay for detection of Zika antibody and (2) willingness to participate. This approach provides a platform to evaluate the suitability of the standard to harmonize as wide a range of assay types as possible, which is essential for robustness of WHO standards. The participants were from six countries: Germany (1), UK (2), Korea (1), Trinidad and Tobago (1), Denmark (1), and USA (13). All laboratories are referred to by a code number allocated at random and are not reflected in the order of listing at the end of the paper. Participating labs included 11 government research, public health, medical counter-measure, and regulatory organizations; three university and research organizations; five developers of biologics, assays, and reagents.

\section{Samples}

The project was approved by the NIBSC Human Material Advisory Committee (project 16/005MP). Plasma and sera were donated to NIBSC anonymized by the organizations listed in Table 2 . All patients signed an informed consent to the use of their sera/plasma.

Collaborative study samples were provided to the participants blind coded and are listed in Table 2. Four pooled samples of plasma (sample S14) or serum (samples S48, S61 and S80) from individuals who were designated as having been infected by ZIKV were prepared at NIBSC, filled in $0.25 \mathrm{~mL} / \mathrm{ampoule}$, freeze-dried and stored at $-20^{\circ} \mathrm{C}$. Prior to filling, two pools, sample S14 and S61, were treated with solvent and detergent ${ }^{40}$ to inactivate blood-borne viruses, as on initial screening these were found to be positive for HIV RNA, HBV antigen or HCV RNA. Post treatment testing for the presence of RNA was negative.

Additional samples were human anti-ZIKV IgG antibodies purified from plasma collected from Tc cattle ${ }^{31}$ immunized with ZIKV immunogens comprising either inactivated virus (Puerto Rico strain cultured in C6/36 insect cells, sample S26), plasmid DNA coding full-length ZIKV E protein (pWRG/Zika PrM-E, sample S1), or the naive control (sample S6). ${ }^{41}$ Upon receipt at NIBSC, the purified Tc bovine IgG samples were diluted to a target protein concentration of $2.5 \mathrm{mg} / \mathrm{mL}$ in sterile phosphate-buffered saline (PBS) $/ \mathrm{Ca}^{2+} \mathrm{Mg}^{2+}$ supplemented with $5 \%$ human serum albumin and aliquoted into $0.1 \mathrm{~mL}$ volumes and stored at $-20^{\circ} \mathrm{C}$.

Negative human plasma and serum were from single donation packs from the UK; all packs were tested for blood borne markers (HIV, HCV, hepatitis B surface antigen and syphilis) and found to be negative.

Anti-DENV serotype antibodies (1-3; serotype 4 was unavailable) were sourced from NIBSC. ${ }^{42}$

\section{Collaborative study assay methods}

Assays used by participants are summarized in Table 1. Where laboratories performed multiple assay methods, laboratory codes are suffixed by a letter indicating the different methods, for example, labs 11a and 11b. The two main assay methods were neutralization of ZIKV, with two laboratories using a RVP system instead of the wild-type virus, and enzyme immunoassays. Anti-ZIKV IgM responses were detected by qualitative assays only, while anti-ZIKV $\lg$ assays were both quantitative and qualitative. SPR was also performed.

\section{NIBSC in-house ELISA}

Nunc Maxisorp 96-well plates were coated overnight at $4{ }^{\circ} \mathrm{C}$ with ZIKV (Asian strain-PRVABC59)-containing supernatant from VERO cells 
diluted in PBS. Conditioned media from uninfected VERO cells was used as a negative control. Virus was fixed with $0.1 \mathrm{~mL}$ per well of $4 \%$ paraformaldehyde diluted in PBS for $30 \mathrm{~min}$ at room temperature. Plates were washed three times with PBS, and then blocked with $0.2 \mathrm{~mL}$ of $10 \%$ foetal bovine serum in PBS for $1 \mathrm{~h}$ at room temperature. Plates were washed three times with PBS containing 0.05\% Tween-20 (PBST). A measure of $0.1 \mathrm{~mL}$ of serum samples diluted $1 / 400$ in blocking buffer were added to the plates in triplicate and incubated at room temperature for 1 hour. Wells were washed with PBST and $0.1 \mathrm{~mL}$ of anti-human horse radish peroxidase conjugate antibody (Jackson ImmunoResearch Inc., cat. no. 109-035-088) diluted 1:5000 in blocking buffer were added to each well. After $1 \mathrm{~h}$ incubation at room temperature, plates were washed with PBST and developed by adding 3,3',5,5'-Tetramethylbenzidine Substrate Systems (Neogen Europe, Ltd.). Reactions were stopped after $10 \mathrm{~min}$ by the addition of $1 \mathrm{M} \mathrm{H}_{2} \mathrm{SO}_{4}$. For the analysis, absorbance from each well was subtracted from the average of absorbance of the diluent-only wells. An optical density $>0.1$ after subtraction with the correspondent negative control was arbitrarily chosen as a positive result.

\section{Study plan}

Participants were requested to test the samples using the method(s) in use in their laboratories for the detection of antibodies to ZIKV. Participants were asked to perform three independent assays on different days. An Excel reporting sheet was provided with suggested dilutions for assaying each study sample. For each assay, participants were requested to make at least two independent series of dilutions of the study samples and assay all samples concurrently if feasible.

\section{Statistical analysis}

For the neutralization assays, the GM of the potency of each sample was calculated from the endpoint titres or $\mathrm{PRNT}_{50}$ provided by the participants. GMs were calculated only when more than half of the results for a sample produced a positive response.

Quantitative ELISA data were analysed using a parallel line model with untransformed or log-transformed responses. Calculations were performed using the European Directorate for the Quality of Medicines \& Healthcare (EDQM) software CombiStats ${ }^{\mathrm{T}}{ }^{43}{ }^{43}$ Model fit was assessed visually, and nonparallelism was assessed by calculation of the ratio of fitted slopes for the test and reference samples under consideration. The samples were concluded to be non-parallel when the slope ratio was outside the range $0.80-1.25$. Relative potency estimates from all valid assays were combined to generate an unweighted GM for each laboratory and assay type, with these laboratory means being used to calculate overall unweighted GMs for each analyte.

Variability between laboratories has been expressed using geometric coefficients of variation $\left(\mathrm{GCV}=\left[10^{s}-1\right] \times 100 \%\right.$, where $s$ is the standard deviation of the $\log _{10}$-transformed estimates). Further assessment of agreement in GM results for each pair of laboratories was performed by calculating Lin's concordance correlation coefficient with log-transformed data, although these values are only based on a small number of samples (between five and seven in all cases). Calculations were performed using the $\mathrm{R}$ package DescTools. ${ }^{44}$

\section{Reporting summary}

Further information on research design is available in the Nature Research Reporting Summary linked to this article.

\section{DATA AVAILABILITY}

Raw data can be made available upon request, but the participant's name will be anonymized. The study samples can also be made available, until depletion of stocks.

Received: 14 March 2019; Accepted: 2 September 2019; Published online: 14 October 2019

\section{REFERENCES}

1. Dick, G. W. Zika virus. II. Pathogenicity and physical properties. Trans. R. Soc. Trop. Med. Hyg. 46, 521-534 (1952).
2. Dick, G. W., Kitchen, S. F. \& Haddow, A. J. Zika virus. I. Isolations and serological specificity. Trans. R. Soc. Trop. Med. Hyg. 46, 509-520 (1952).

3. Duffy, M. R. et al. Zika virus outbreak on Yap Island, Federated States of Micronesia. N. Engl. J. Med. 360, 2536-2543 (2009).

4. Cao-Lormeau, V. M. et al. Guillain-Barre Syndrome outbreak associated with Zika virus infection in French Polynesia: a case-control study. Lancet 387, 1531-1539 (2016).

5. de Araujo, T. V. B. et al. Association between Zika virus infection and microcephaly in Brazil, January to May, 2016: preliminary report of a case-control study. Lancet Infect. Dis. 16, 1356-1363 (2016).

6. Rasmussen, S. A., Jamieson, D. J., Honein, M. A. \& Petersen, L. R. Zika virus and birth defects-reviewing the evidence for causality. N. Engl. J. Med. 374, 1981-1987 (2016).

7. WHO. Zika Situation Report-Neurological Syndrome and Congenital Anomalies. http:// apps.who.int/iris/bitstream/handle/10665/204348/zikasitrep_5Feb2016_eng.pdf; jsessionid=83509CB6FF8F02E53DCEB101F33772A0? sequence $=1$ (2016).

8. WHO. Fifth Meeting of the Emergency Committee under the International Health Regulations (2005) Regarding Microcephaly, Other Neurological Disorders and Zika Virus. https://www.who.int/news-room/detail/18-11-2016-fifth-meeting-of-the-emerg ency-committee-under-the-international-health-regulations-(2005)-regarding-microc ephaly-other-neurological-disorders-and-zika-virus (2016).

9. WHO. Zika Vaccine Development Technology Roadmap. https://www.who.int/ immunization/research/development/Zika_Vaccine_Development_Technology_ Roadmap_final_2018.pdf (2018).

10. WHO/UNICEF. WHO/UNICEF Zika Virus (ZIKV) Vaccine Target Product Profile (TPP): Vaccine to Protect Against Congenital Zika Syndrome For Use During An Emergency. https://www.who.int/immunization/research/development/WHO_UNICEF_Zikavac_ TPP_Feb2017.pdf?ua=1 (2017).

11. Barrett, A. D. T. Current status of Zika vaccine development: Zika vaccines advance into clinical evaluation. npj Vaccines 3, 24 (2018).

12. CDC. Testing for Zika. https://www.cdc.gov/zika/symptoms/diagnosis.html (2019).

13. WHO. Laboratory Testing for Zika Virus Infection. http://apps.who.int/iris/ bitstream/handle/10665/204671/WHO_ZIKV_LAB_16.1_eng.pdf?sequence=1 (2016).

14. Foy, B. D. et al. Probable non-vector-borne transmission of Zika virus, Colorado, USA. Emerg. Infect. Dis. 17, 880-882 (2011).

15. Musso, D. et al. Potential sexual transmission of Zika virus. Emerg. Infect. Dis. 21, 359-361 (2015).

16. Paz-Bailey, G. et al. Persistence of Zika virus in body fluids-final report. N. Engl. J. Med. 379, 1234-1243 (2018).

17. CDC. Diagnostic Tests for Zika Virus. https://www.cdc.gov/zika/hc-providers/typesof-tests.html (2018).

18. Gourinat, A. C., O'Connor, O., Calvez, E., Goarant, C. \& Dupont-Rouzeyrol, M. Detection of Zika virus in urine. Emerg. Infect. Dis. 21, 84-86 (2015).

19. Lanciotti, R. S. et al. Genetic and serologic properties of Zika virus associated with an epidemic, Yap State, Micronesia, 2007. Emerg. Infect. Dis. 14, 1232-1239 (2008).

20. Martin, D. A. et al. Standardization of immunoglobulin $M$ capture enzyme-linked immunosorbent assays for routine diagnosis of arboviral infections. J. Clin. Microbiol. 38, 1823-1826 (2000).

21. Rabe, I. B. et al. Interim guidance for interpretation of Zika virus antibody test results. Morb. Mortal. Wkly Rep. 65, 543-546 (2016).

22. Priyamvada, L., Suthar, M. S., Ahmed, R. \& Wrammert, J. Humoral immune responses against Zika virus infection and the importance of preexisting flavivirus immunity. J. Infect. Dis. 216, S906-S911 (2017).

23. Balmaseda, A. et al. Antibody-based assay discriminates Zika virus infection from other flaviviruses. Proc. Natl. Acad. Sci. USA 114, 8384-8389 (2017).

24. Steinhagen, K. et al. Serodiagnosis of Zika virus (ZIKV) infections by a novel NS1based ELISA devoid of cross-reactivity with dengue virus antibodies: a multicohort study of assay performance, 2015 to 2016. Euro Surveill. 21, https://doi. org/10.2807/1560-7917.ES.2016.21.50.30426 (2016).

25. Vannice K. S. et al. Demonstrating vaccine effectiveness during a waning epidemic: a WHO/NIH meeting report on approaches to development and licensure of Zika vaccine candidates. Vaccine, https://doi.org/10.1016/j.vaccine.2018.12.040 (2019).

26. Wilder-Smith, A. et al. Zika vaccines and therapeutics: landscape analysis and challenges ahead. BMC Med. 16, 84 (2018).

27. WHO. Blood Product and Related Biologicals_International Reference Materials. https://www.who.int/bloodproducts/ref_materials/en/ (2018).

28. Mukherjee, R. et al. Diagnosis and management of hepatitis C virus infection. J. Lab Autom. 20, 519-538 (2015).

29. WHO. Requirements for yellow fever vaccine (requirements for biological substances no. 3). In WHO Expert Committee on Biological Standardization, Forty-Sixth Report WHO Technical Report Series No. 872 (World Health Organization, Geneva, 1998). 
30. Ferguson, M. \& Heath, A. Collaborative study to assess the suitability of a candidate International Standard for yellow fever vaccine. Biologicals 32, 195-205 (2004).

31. Kuroiwa, Y. et al. Antigen-specific human polyclonal antibodies from hyperimmunized cattle. Nat. Biotechnol. 27, 173-181 (2009).

32. Wilkinson, D. E. et al. WHO collaborative study to assess the suitability of the 1st International Standard and the 1st International Reference Panel for antibodies to Ebola virus. WHO/BS/2017.2316 (2017).

33. Anfasa, F. et al. Phenotypic differences between Asian and African lineage Zika viruses in human neural progenitor cells. mSphere 2, https://doi.org/10.1128/ mSphere.00292-17 (2017).

34. Dowall, S. D. et al. Lineage-dependent differences in the disease progression of Zika virus infection in type-I interferon receptor knockout (A129) mice. PLoS Negl. Trop. Dis. 11, e0005704 (2017).

35. Simonin, Y. et al. Zika virus strains potentially display different infectious profiles in human neural cells. EBioMedicine 12, 161-169 (2016).

36. Tripathi, S. et al. A novel Zika virus mouse model reveals strain specific differences in virus pathogenesis and host inflammatory immune responses. PLoS Pathog. 13, e1006258 (2017).

37. Dowd, K. A. et al. Broadly neutralizing activity of Zika virus-immune sera identifies a single viral serotype. Cell Rep. 16, 1485-1491 (2016).

38. Montoya, M. et al. Longitudinal analysis of antibody cross-neutralization following Zika virus and dengue virus infection in Asia and the Americas. J. Infect. Dis. 218, 536-545 (2018)

39. WHO. Expert Committee on Biological Standardization 69th Technical Report Series no. 1016 (World Health Organisation, Geneva, 2019).

40. Dianna, E. W. et al. WHO collaborative study to assess the suitability of an interim standard for antibodies to Ebola virus. WHO/BS/2015.2280 post-ECBS (2015).

41. Stein, D. R. et al. Human polyclonal antibodies produced in transchromosomal cattle prevent lethal Zika virus infection and testicular atrophy in mice. Antivir. Res. 146, 164-173 (2017).

42. Ferguson, M., Johnes, S. \& Heath, A. Report of a collaborative study to assess the suitability of candidate reference material to serve as the first WHO reference reagent dengue virus antibody and to standardize the neutralization test for dengue viruses. WHO/BS/05.2209 (2005).

43. EDQM. CombiStats, https://www.edqm.eu/combistats/ (2013).

44. Andri, S. DescTools: Tools for Descriptive Statistics, https://cran.r-project.org/ package $=$ DescTools (2018)

\section{ACKNOWLEDGEMENTS}

We gratefully acknowledge the important contributions of the collaborative study participants. We would also like to thank NIBSC Standards Production and Development for distribution of the candidate materials. We also thank David Wood and Micha Nuebling of the WHO and participants of teleconferences for their support, guidance and advice, and Steven A. Rubin, Swati Verma, Hira Nakhasi, and Uwe Scherf FDA/CBER, USA for facilitating the sample permits and shipments to laboratories in the USA. This paper is based on independent research commissioned and funded by the NIHR Policy Research Programme (NIBSC Regulatory Science Research Unit). The views expressed in the publication are those of the author(s) and not necessarily those of the NHS, the NIHR, the Department of Health, 'arms' length bodies or other government departments.

\section{AUTHOR CONTRIBUTIONS}

M.P., D.E.W. and G.M. designed the study plan. M.H., J.A., S.M. and V.F. characterized and formulated the samples and performed the stability studies. J.H. and P.R. performed the statistical analysis. Collaborative study participants produced the data. G.M., I.K. and M.P. wrote the manuscript.

\section{COMPETING INTERESTS}

The authors declare no competing interests.

\section{ADDITIONAL INFORMATION}

Supplementary information is available for this paper at https://doi.org/10.1038/ s41541-019-0135-3.

Correspondence and requests for materials should be addressed to G.M.

Reprints and permission information is available at http://www.nature.com/ reprints

Publisher's note Springer Nature remains neutral with regard to jurisdictional claims in published maps and institutional affiliations.

\begin{abstract}
Open Access This article is licensed under a Creative Commons Attribution 4.0 International License, which permits use, sharing, adaptation, distribution and reproduction in any medium or format, as long as you give appropriate credit to the original author(s) and the source, provide a link to the Creative Commons license, and indicate if changes were made. The images or other third party material in this article are included in the article's Creative Commons license, unless indicated otherwise in a credit line to the material. If material is not included in the article's Creative Commons license and your intended use is not permitted by statutory regulation or exceeds the permitted use, you will need to obtain permission directly from the copyright holder. To view a copy of this license, visit http://creativecommons. org/licenses/by/4.0/.
\end{abstract}

(c) Crown 2019

\section{THE COLLABORATIVE STUDY PARTICIPANTS}

Marco Donolato ${ }^{4}$, Sally Baylis ${ }^{5}$, Constanza Yue ${ }^{5}$, Fabian Elgner ${ }^{5}$, In-Kyu Yoon ${ }^{6}$, Jae Seung Yang ${ }^{6}$, Manki Song ${ }^{6}$, Gabriel GonzalezEscobar ${ }^{7}$, Richard Brindle ${ }^{7}$, Richard Tedder ${ }^{8}$, Steve Dicks ${ }^{8}$, Ines Ushiro-Lumb ${ }^{8}$, Sarah Williams-McDonald ${ }^{1}$, Sanjai Kumar $^{9}$, Keith Peden $^{9}$, Hana Golding ${ }^{9}$, Surender Khurana ${ }^{9}$, Matthew Bonaparte ${ }^{10}$, Anna Durbin ${ }^{11}$, Hansi Dean ${ }^{12}$, Stephanie Sonnberg ${ }^{12}$, Wayne Hogrefe ${ }^{13}$, James Crowe ${ }^{14}$, Thomas Voss ${ }^{14}$, Matthew Collins ${ }^{15}$, Theodore Pierson ${ }^{16}$, Katherine Burgomaster ${ }^{16}$, Kimberly Dowd ${ }^{16}$, Louise Sigismondi ${ }^{17}$, Dhammika Gunasekera ${ }^{17}$, James Wassenberg ${ }^{18}$, Kenneth Eckels ${ }^{19}$ and Rafael De La Barrera ${ }^{19}$

\footnotetext{
${ }^{4}$ BlueSense Diagnostics ApS, Copenhagen, Denmark. ${ }^{5}$ Paul-Ehrlich-Institut, Langen, Germany. ${ }^{6}$ International Vaccine Institute, Seoul, Korea. ${ }^{7}$ Caribbean Public Health Agency, St. Clair- Port of Spain, Trinidad and Tobago. ${ }^{8}$ Virus Reference Department, Public Health England, London, UK. ${ }^{9}$ Center for Biologics Evaluation and Research, US Food and Drug Administration, Bethesda, MD, USA. ${ }^{10}$ Sanofi Pasteur, Swiftwater, PA, USA. ${ }^{11}$ Johns Hopkins Bloomberg School of Public Health, Baltimore, MD, USA. ${ }^{12}$ Takeda Vaccines Inc., Deerfield, IL, USA. ${ }^{13}$ Q2 Solutions-Vaccines, Focus Diagnostics Inc., San Juan Capistrano, CA, USA. ${ }^{14}$ Vanderbilt University Medical Center, Nashville, TN, USA. ${ }^{15}$ University of North

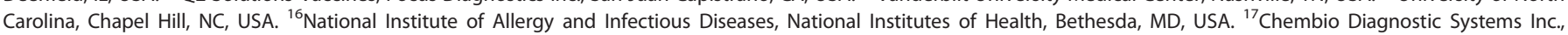
Medford, NY, USA. ${ }^{18}$ DiaSorin Inc., Stillwater, MN, USA. ${ }^{19}$ Walter Reed Army Institute of Research, Silver Spring, MD, USA
} 